\title{
HARMONIC-SUPPRESSED MINIATURIZED-ELEMENT FREQUENCY SELECTIVE SURFACES WITH HIGHER-ORDER BANDPASS RESPONSES
}

by

Seyed Mohamad Amin Momeni Hasan Abadi

\begin{abstract}
A masters project report submitted in partial fulfillment of the requirements for the degree of

Master of Science

(Electrical Engineering)
\end{abstract}

at the

UNIVERSITY OF WISCONSIN-MADISON 
(c) Copyright by Seyed Mohamad Amin Momeni Hasan Abadi 2013

All Rights Reserved 


\section{ABSTRACT}

In this reprot, we introduce a new technique for designing miniaturized-element frequency selective surfaces (MEFSSs) with bandpass responses and no spurious transmission windows over extremely large bandwidths. The proposed, harmonic-suppressed MEFSSs consist of multiple metallic and dielectric layers. Each metallic layer is in the form of a two-dimensional arrangement of capacitive patches or an inductive wire grid with extremely sub-wavelength periods. Harmonicfree operation in these structures is achieved by using multiple, closely-spaced capacitive layers with overlapping unit cells to synthesize a single, effective capacitive layer with a larger capacitance value. This allows for reducing the unit cell size of a conventional MEFSS considerably and moving the natural resonant frequencies of its constituting elements to considerably higher frequencies. Consequently, the spurious transmission windows of such MEFSSs, which are caused by these higher-order harmonics, can be shifted to very high frequencies and an extremely broad frequency band free of any spurious transmission windows can be obtained. Using this technique, an MEFSS with a second-order bandpass response is designed to operate at $3.0 \mathrm{GHz}$ with $20 \%$ fractional bandwidth and be free of spurious transmission bands up to $27.0 \mathrm{GHz}$. A prototype of this harmonic-free MEFSS is fabricated and experimentally characterized in the lab. Measurement results confirm harmonic-free operation of the proposed FSS for incidence angles in the $\pm 60^{\circ}$ range for both the TE and TM polarizations of incidence. 


\section{Chapter 1}

\section{Introduction}

Frequency selective surfaces (FSSs) have been the subject of numerous studies so far. At microwave and millimeter-wave frequencies, FSSs are used in a wide range of applications including spatial filters [1], [2], radar absorbing materials [3], [4], artificial magnetic conductors [5], [6], planar lenses [7], [8] and reflectarrays [9]. One common application of FSSs is to use them to reduce the radar cross section (RCS) of antennas used in low-observable or stealth platforms. Most antennas act as efficient scatterers both at the frequency band(s) that they are designed to operate in and at other frequency bands that fall outside of their desired frequency range of operation. A typical low-observable platform may have several low-frequency antennas operating at VHF and UHF bands. These antennas act as very efficient scatterers at higher frequencies where many radars operate at. In such applications, the RCS of the antenna can be reduced by shielding it from the outside environment using a shaped bandpass FSS that is transparent within the desired frequency of operation of the antenna and opaque at other frequencies. While the aforementioned RCS reduction approach works in principle, it suffers from a practical design problem. Namely, most FSSs reported in the literature have multiple spurious transmission windows occurring at frequencies higher than that of the main one (e.g. see pp. 26-62 of [1]). Although the high frequency harmonics typically will not impact the in-band performance of these FSSs, they could become critically important in certain stealth applications in which they coincide with a frequency at which the an-

tenna presents a high RCS value. For an FSS designed to work at a low frequency (e.g. UHF), a number of these spurious transmission windows can fall within the 1-20 GHz range where many radars operate. Thus, in such applications, suppression of the spurious harmonics of the FSS is highly desired. 
A number of previous studies has been conducted in this area [10]-[12]. In [10], an absorptive/transmissive radome is presented. The presented multilayer structure is formed by placing an artificial absorbing coating on a frequency selective surface. This structure, however, achieves absorption properties by using a uniform resistive layer of conductive fiber, which deteriorates the in-band performance of the radome as well. In [11], a bandpass FSS with quasi-elliptic response and wide-stopband characteristics is reported. The structure is formed by cascading periodic arrays of double square loops and gridded square loops. However, this approach requires using several metallic layers separated from one another by rather thick dielectric substrates to design a lowfrequency (e.g. UHF) FSS. This leads to a relatively large overall thickness for the FSS (compared to the wavelength) that makes the response of the structure sensitive to the angle and polarization of incidence of the EM wave. In [12], a bandpass FSS with wideband absorbing properties is presented. The reported structure behaves as a bandpass filter in the main band of operation, while behaving as an absorber above the main band. This is realized by cascading a bandpass FSS and a resistive high-impedance surface which uses the FSS as the ground plane within its total reflection band. Although a relatively good performance was observed under normal incidence of the EM wave, the performance of this structure degrades when the structure is illuminated with an obliquely incident wave. The problem can be attributed to the absorption properties of the resistive surface which degrade as a function of the incident angle. As a result, some harmonics appear within the absorption band. This is not desirable in low-observable FSS and radome applications.

Over the past few years, several new classes of frequency selective surfaces with sub-wavelength unit cells dimensions are reported [13]-[24]. These structures are referred to as miniaturized element frequency selective surfaces. Depending on the specific design process, MEFSSs are composed of entirely non-resonant constituting elements (e.g., [13]-[18], [21]) or a combination of resonant and non-resonant elements (e.g. [19]-[20], [22]-[24]). MEFSSs are shown to have very stable responses for a wide range of incidence angles and polarizations of incidence [13]-[21]. They also have been used to design tunable FSSs [17], [25] as well as FSSs for high-power microwave (HPM) applications [25]-[26]. 
In this report, we present a new type of MEFSS that shows a bandpass response, which is free of spurious transmission windows over an extremely wide frequency band. The proposed harmonic-free MEFSS is composed of multiple metallic layers separated from one another by thin dielectric substrates. Each metallic layer is a two-dimensional periodic structure composed of capacitive patches or inductive wire grids with extreme sub-wavelength dimensions. A prototype of the proposed harmonic-free MEFSS with a second-order bandpass response, center frequency of operation of $3.0 \mathrm{GHz}$, and fractional bandwidth of $20 \%$ is designed and is shown to be harmonic free up to approximately $27 \mathrm{GHz}$. A prototype of the proposed structure is also fabricated and experimentally characterized in the lab. It is demonstrated that the proposed structure maintains its harmonic-free, second-order bandpass response for both the TE and TM polarizations with incidence angles in the $\pm 60^{\circ}$ range up to $27 \mathrm{GHz}$. 


\subsection{Principles of Operation}

\subsubsection{Sources of Harmonics in MEFSSs}

Fig. 1.1(a) shows the three-dimensional topology of different layers of a miniaturized element frequency selective surface (MEFSS) with an $\left(\frac{N+1}{2}\right)^{t h}$-order bandpass response ( $N$ is the number of metallic layers used in this structure and is always an odd number [21]). The structure consists of two-dimensional periodic arrangements of sub-wavelength capacitive patches and planar wire grids, separated from one another by thin dielectric substrates. Assuming that the thickness of the substrate between two consecutive metal layers is $h$, the overall thickness of the FSS is $(N-1) \times h$. The top views of one unit cell of a capacitive layer and that of an inductive layer are shown in the inset of Fig. 1.1(a). The dimension of each unit cell along the $x$ and $y$ directions is $D$. The capacitive patches are in the form of square metallic patches with dimensions of $D-s_{i}$, where $s_{i}$ is the gap between the two adjacent patches in the $i^{t h}$ layer. The inductive wire grids are the combination of two metallic strips with the width of $w$ oriented perpendicularly to each other. Assuming the same periodicity in both $x$ and $y$ directions, the frequency responses of the capacitive and inductive layers (and that of the FSS) are insensitive to the polarization of the incident wave for normal incidence.

This FSS can be modeled with the equivalent circuit model shown in Fig. 1.1(b), which is valid for a normally incident plane wave. In this circuit model, the capacitive patch layers are modeled with parallel capacitors $C_{1}, C_{3}, \ldots, C_{N}$. The inductive wire grids are represented by parallel inductors $L_{2}, \ldots, L_{N-1}$, and the thin dielectric substrates separating the inductive and capacitive layers are modeled with transmission lines with the characteristic impedances of $Z_{1}, \ldots, Z_{N-1}$ and lengths of $h_{1}, \ldots, h_{N-1}$. Free space on each side of the FSS is modeled with semi-infinite transmission lines with characteristic impedances of $Z_{\circ}=377 \Omega$. The design procedure of this device is based on synthesizing the desired filter response from the equivalent circuit model presented in Fig. 1.1(b), and mapping these equivalent circuit parameter values to the physical parameters of the FSS. This can be done using the procedure described in [21] and will not be repeated here for brevity. 


\begin{tabular}{|c||c|c|c|c|}
\hline Parameter & $D_{x}$ & $D_{y}$ & $s$ & $w$ \\
\hline Value & $24 \mathrm{~mm}$ & $24 \mathrm{~mm}$ & $0.35 \mathrm{~mm}$ & $11 \mathrm{~mm}$ \\
\hline Parameter & $h$ & $C_{1}$ & $L_{2}$ & $C_{3}$ \\
\hline Value & $1.575 \mathrm{~mm}$ & $1.18 \mathrm{pF}$ & $0.34 \mathrm{nH}$ & $1.18 \mathrm{pF}$ \\
\hline
\end{tabular}

Table 1.1 Physical and electrical parameters of the $2^{\text {nd }}$-order MEFSS with a center frequency of 3 $\mathrm{GHz}$ and the fractional bandwidth of $\delta=20 \%$ discussed in Section 1.1.1.

Using the design procedure reported in [21], an MEFSS with a second-order bandpass response having a center frequency of operation of $3.0 \mathrm{GHz}$ and a fractional bandwidth of $20 \%$ is designed. This structure is composed of two capacitive layers, one inductive layer, and two dielectric substrates. The physical parameters of this structure as well as the parameters of its equivalent circuit model are presented in Table 1.1. This MEFSS is simulated using full-wave electromagnetic (EM) simulations in CST Microwave Studio and its frequency response is calculated over an extremely broad frequency band. Fig. 1.2 shows the calculated transmission coefficient of this structure for a normally-incident plane wave in the frequency range of 0-30 GHz. As can be seen, in addition to the main transmission band centered at $3.0 \mathrm{GHz}$, there are multiple spurious transmission windows occurring at higher frequencies than that of the main one. The primary contributors to these harmonics are the resonances of the patches in the capacitive layers and the slots in the inductive layer that occur at higher frequencies. For example, the frequency of the first resonance of the capacitive patches in the capacitive layer and the slots in the inductive layer can be approximated using (1)-(2):

$$
\begin{aligned}
f_{r, \text { cap }} & =\frac{c}{2(D-s) \sqrt{\epsilon_{r, e f f, i}}} \\
f_{r, \text { ind }} & =\frac{c}{2(D-w) \sqrt{\epsilon_{r, e f f, i}}}
\end{aligned}
$$

where $D, s_{i}$, and $w_{i}$ are the physical parameters of the FSS unit cell shown in Fig. 1.1, $c$ is the speed of light, and $\epsilon_{r, e f f, i}$ is the effective dielectric constant for the $i^{t h}$ metallic layer of the FSS. 


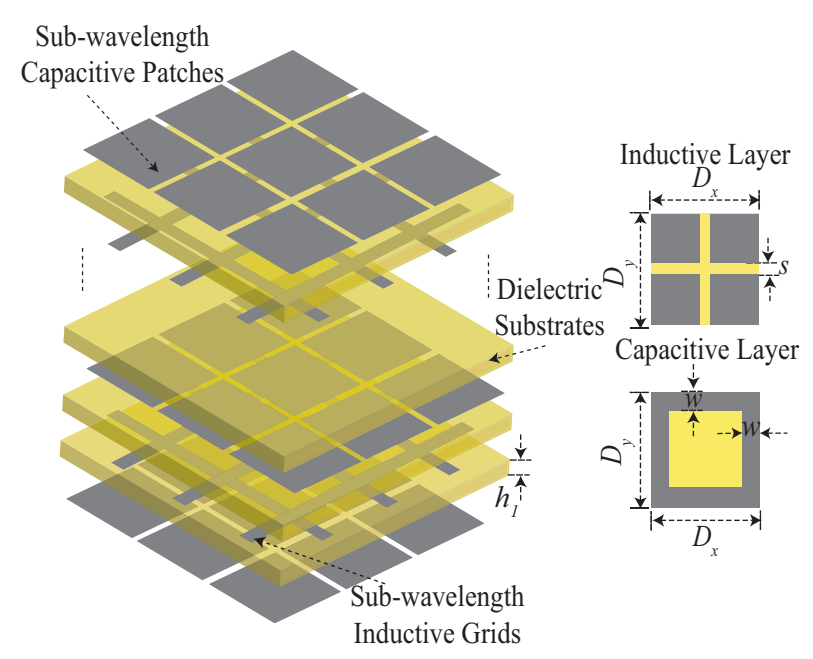

(a)

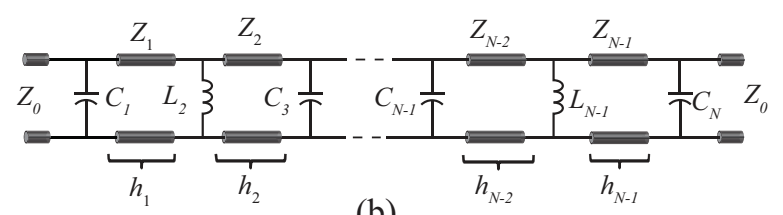

(b)

Figure 1.1 (a) Topology of the bandpass MEFSS presented in [21]. The top view of the unit cells of the capacitive and inductive layers are shown on the right hand side of the figure. (b) Equivalent circuit model of the MEFSS. The structure has $N$ metal layers and acts as an FSS with an $\left(\frac{N+1}{2}\right)^{t h}$-order bandpass response.

In the second-order MEFSS example under discussion in this section, $\epsilon_{r, e f f, 1}=\epsilon_{r, e f f, 3} \approx \frac{\epsilon_{r}+1}{2}$ for the capacitive layers and $\epsilon_{r, e f f, 2} \approx \epsilon_{r}$ for the inductive layer. Notice that in (1)-(2) the effect of the interaction between different layers on the higher-order resonances of the constituting elements in each unit cell is ignored. Therefore, these formulas represent approximations of the actual resonant frequencies of the capacitive patches and apertures in the inductive layers. Using (1)-(2) along with the physical parameters of the MEFSS provided in Table 1.1, the first resonant frequencies of the capacitive patches and the slots in the inductive layers are calculated to be $5.0 \mathrm{GHz}$ and 7.8 $\mathrm{GHz}$, respectively. At their first resonance, the patches in the capacitive layers act as band-stop elements, whereas the apertures in the inductive layer act as bandpass elements (see pp. 26-62 of [1]). Therefore, the first spurious transmission window of this second-order MEFSS is expected to occur at a frequency close to the resonant frequency of the apertures in the inductive layer. Fig. 


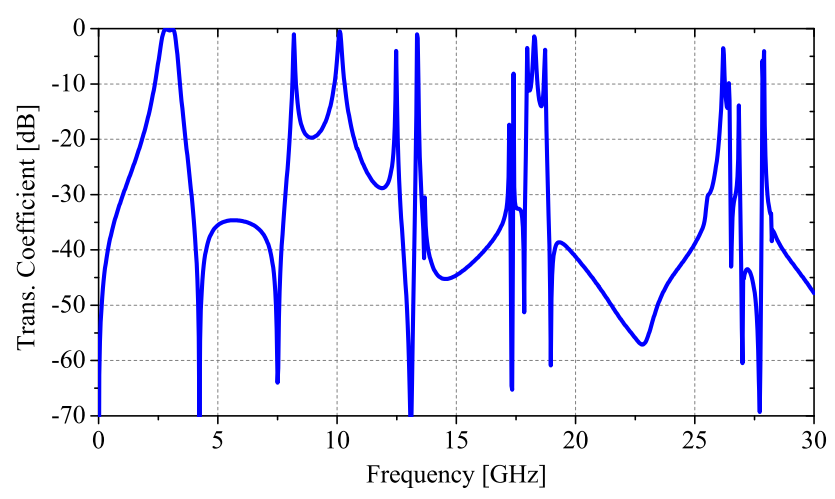

Figure 1.2 Calculated transmission coefficient of the $2^{\text {nd }}$-order bandpass FSS discussed in Section 1.1.1 with the parameters shown in Table 1.1. Results are obtained using full-wave EM simulation in CST Microwave Studio.

1.2 shows that this is indeed the case. At higher frequencies, the higher-order resonant modes of the elements, the complex interactions between different layers, as well as the grid resonances occurring at $D=n \lambda$ (e.g. see pp. 26-62 of [1]) result in additional transmission windows and transmission nulls in the transmission coefficient of this device as can be observed in Fig. 1.2.

\subsubsection{A Concept for Designing Harmonic-Free MEFSSs}

Since the first spurious passband of this MEFSS is caused by the natural harmonics of its constituting elements, an effective method for removing that from the desired frequency band of operation is to reduce the unit cell size of the structure. From (1)-(2), it can be seen that reducing $D$ shifts these resonant frequencies to higher values. In doing this, however, the effective inductance and capacitance values provided from the metallic layers of the MEFSS must not change in order to maintain the frequency response of the structure within the desired transmission band (i.e., the element values of the equivalent circuit model of the MEFSS must not change). This shifts the spurious transmission bands to higher frequencies without disturbing the frequency response of the FSS within the desired operating band. However, implementing this in practice can be challenging, since reducing the period of the MEFSS while maintaining the desired capacitance and inductance values results in significant reduction of the minimum feature sizes of the unit cell of the MEFSS (i.e., the gap size between capacitive patches $s_{i}$ and the width of the inductive wires $w_{i}$ ) [21]. For 


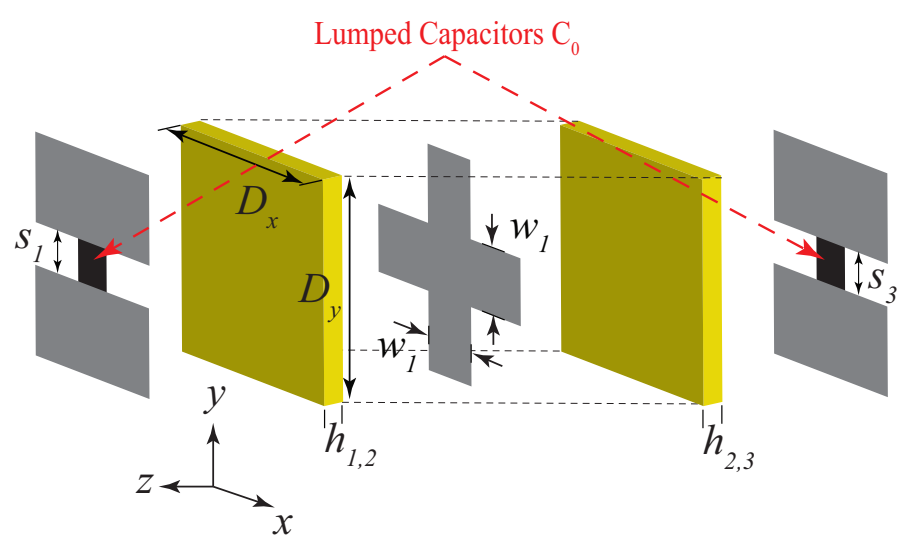

Figure 1.3 The unit cell of the $2^{\text {nd }}$-order bandpass MEFSS with the lumped capacitors loading the capacitive patch layers.

MEFSSs of the type shown in Fig. 1, however, this problem is more severe for the capacitive patch layers and the gap spacing between adjacent capacitive patches in these layers is the bottleneck in the process of miniaturization of the MEFSS unit cell [16]. In practice, the minimum spacing between the adjacent capacitive patches in each capacitive layer of the FSS, $s_{i}$, is determined by the minimum feature size that can be reliably fabricated using any given fabrication procedure. For most standard lithography techniques used in printed circuit board (PCB) fabrication, this minimum feature size is about $0.15 \mathrm{~mm}$. Therefore, achieving the desired capacitance value from an extremely sub-wavelength periodic structure is rather challenging.

One technique for achieving the desired capacitance value in such situations is shown in Fig. 1.3. In this case, lumped-element capacitors $C_{0}$ are placed in parallel between the two adjacent edges of nearby capacitive patches. Taking the intrinsic capacitance of the unloaded capacitive patch layer, $C_{i}$, into account, the total capacitance of each capacitive layer in this structure can be modeled with a parallel combination of $C_{0}$ and $C_{i}$. Using this topology, the unit cell size of the capacitive patches of the structure can be reduced while maintaining the required capacitance value. To demonstrate this in practice, three different MEFSSs of the type shown in Fig. 1.3 are designed and simulated. All of these MEFSSs have second-order bandpass responses centered at $3.0 \mathrm{GHz}$ and a fractional bandwidth of $20 \%$. For all three cases, the gap sizes in the capacitive layers are $s=s_{1}=s_{3}=0.35 \mathrm{~mm}$, and the thicknesses of the substrates are $h_{1,2}=h_{2,3}=1.575 \mathrm{~mm}$. 


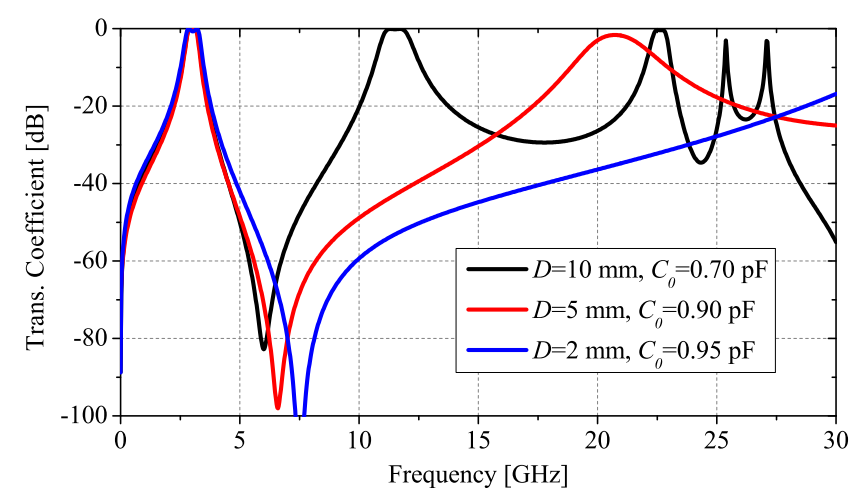

Figure 1.4 Calculated transmission coefficients of the $2^{\text {nd }}$-order bandpass MEFSS discussed in Section 1.1.2 where the capacitive patches are loaded with lumped-element capacitors. Results are obtained using full-wave EM simulation in CST Microwave Studio.

However, these MEFSSs have different periods and consequently, use different lumped-element capacitor values to maintain the desired frequency response. Fig. 1.4 shows the transmission coefficients of these three MEFSSs obtained using full-wave EM simulations in CST Studio. As expected, the frequencies of the spurious transmission windows increase as the unit cell sizes of the MEFSSs decrease. For example, for the structure with the unit cell size of $D=10 \mathrm{~mm}$, and the lumped capacitor value of $C_{0}=0.7 \mathrm{pF}$, the first spurious transmission window occurs at 11.5 GHz. As the unit cell size is decreased (and $C_{0}$ is increased), the frequency of the first spurious transmission window increases as well and in the extreme case of $D=2 \mathrm{~mm}$ and $C_{0}=0.95 \mathrm{pF}$, the first spurious transmission window occurs at a frequency above $30 \mathrm{GHz}$.

\subsubsection{Practical Implementation of Harmonic-Free MEFSSs}

While this design example confirms the feasibility of removing spurious transmission bands from the responses of MEFSSs over a very wide frequency range, a major challenge limits the practicality of implementing MEFSSs of the type shown in Fig. 1.3. Specifically, the number of lumped elements needed to achieve the desired response can become extremely large, even in small FSS panels. For example, for $D=2 \mathrm{~mm}$, a $10 \mathrm{~cm} \times 10 \mathrm{~cm}$ panel of the second-order MEFSS whose response is shown in Fig. 1.4 requires about 10,000 lumped capacitors ( 2 capacitive layers, 2500 capacitive patches per layer, and 2 lumped capacitors per patch to ensure polarization 


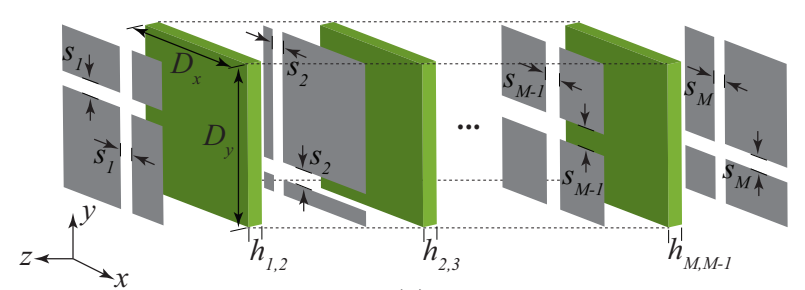

(a)

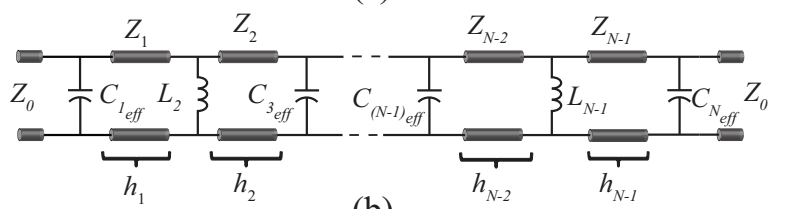

(b)

Figure 1.5 (a) Multiple closely-spaced capacitive patch layers are used to synthesize a single effective capacitive layer. The dielectric substrates are extremely thin. The unit cells of the patches used in different layers can overlap with each other and do not necessarily have to be aligned together. (b) The equivalent circuit model of a generalized MEFSS of the type shown in Fig. 1.1 in which each one of the capacitive layers is substituted with a composite, multi-layer effective capacitive layer similar to that shown in Fig. 1.5(a). In this equivalent circuit model $C_{i, e f f}$ represents the effective capacitance of each of the composite, multi-layer capacitive structures that are used to synthesize the $i^{t h}$ capacitive layer of the MEFSS shown in Fig. 1.1(a).

insensitivity). This becomes even more challenging when a higher-order filter response is required, since the number of capacitive layers in these filters increases [21]. Additionally, in MEFSSs with bandpass responses of order $P \geq 3, P-2$ capacitive layers are sandwiched by dielectric substrates on both sides and are not directly accessible [21].

While the direct implementation of the MEFSS shown in Fig. 1.3 using lumped elements is challenging, alternative implementation techniques can be envisioned that address the practical problems of using lumped elements in a periodic structure. One such technique is to use cascaded capacitive patches separated by very thin dielectric substrates to synthesize a single capacitive layer with an effectively larger capacitance. In such an arrangement, each capacitive layer of the original MEFSS shown in Fig. 1.1 is replaced with multiple, closely-spaced capacitive patch layers. In general, as the period of the MEFSS is reduced, the number of closely-spaced capacitive patch layers needed to synthesize an effective capacitance layer will increase. Fig. 1.5(a) shows the unit cell of an effective capacitive layer composed of $M$ individual capacitive patches separated from one another by $M-1$ extremely thin dielectric substrates. For small substrate thicknesses, all these 
capacitive patches can be considered to be in parallel with one another and hence, they constitute a single composite capacitive layer with a larger capacitance value. Additionally, different capacitive patch layers that constitute the composite capacitive layer can overlap with each other to further increase the capacitance of the composite structure. Using $M$ closely-spaced capacitive patch layers to implement each single capacitive layer of the MEFSS shown in Fig. 1.1 will increase the total number of metallic layers of the MEFSS to $M \times P+P-1$, where $P=\frac{N+1}{2}$ is the order of the filter, and $N$ is the total number of metallic layers of the original MEFSS shown in Fig. 1.1. The equivalent circuit model of the whole structure is shown in Fig. 1.5(b). Here, the combination of cascaded capacitive patches are modeled with $C_{1_{e f f}}, \ldots, C_{N_{e f f}}$. Each capacitor in this equivalent circuit represents the effective capacitance obtained from one combination of $M$ closely-spaced capacitive layers. The sub-wavelength inductive wiregrids are modeled with $L_{2}, \ldots, L_{N-1}$ and the dielectric substrates separating the inductive layers and combined capacitive layers are modeled with the transmission lines with the lengths of $h_{1}, \ldots, h_{N-1}$ and the characteristic impedances of $Z_{1}, \ldots, Z_{N-1}$. Free space on each side of the FSS is modeled with semi-infinite transmission lines with the characteristic impedances of $Z_{\circ}=377 \Omega$.

There are a number of parameters that influence the effective capacitance obtained from the combination of cascaded patches. These include the number of capacitive layers in the cascade arrangement, the gap size between the adjacent capacitive patches in each layer, and the thickness and the dielectric constants of the separating substrates. Increasing the number of layers increases the effective capacitance but it will also increase the total number of layers used in the MEFSS and increases the overall thickness and the complexity of the design. Therefore, in the rest of this paper, we focus on designs that use only two cascaded capacitive layers to synthesize each of the constituting capacitive layers of the MEFSS of Fig. 1.1. Decreasing the gap size, increasing the dielectric constant value of the separating substrates, and decreasing their thicknesses will all increase the effective capacitance value. However, these parameters are primarily determined by the fabrication technology or the availability of commercialy available substrates. In addition to these parameters, the capacitance value also depends on the offset overlap between two cascaded patches in the capacitive layer. 


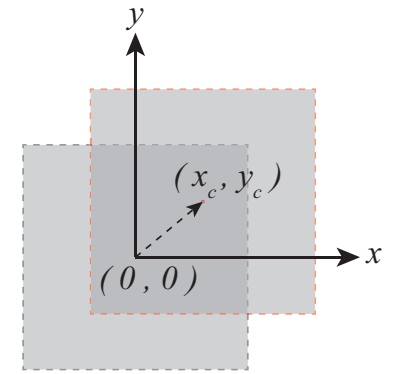

(a)

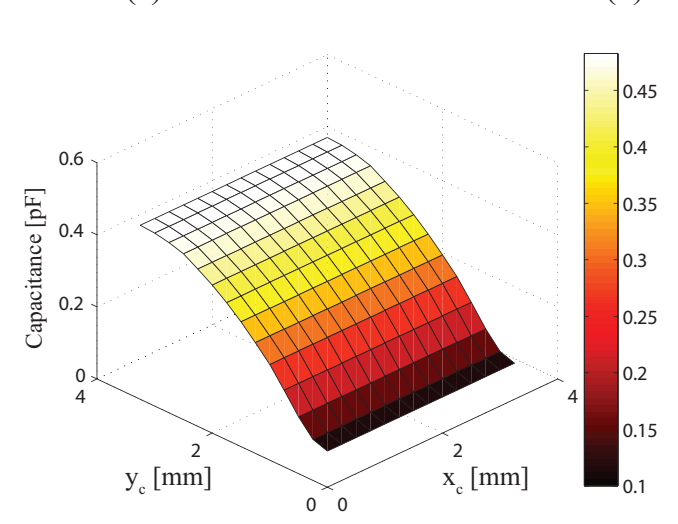

(d)

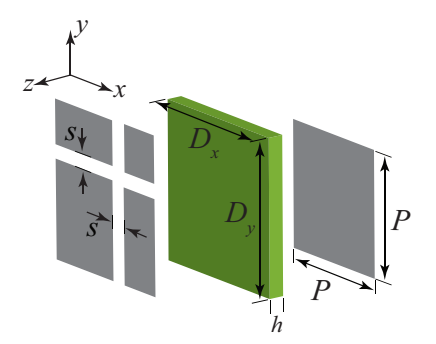

(b)

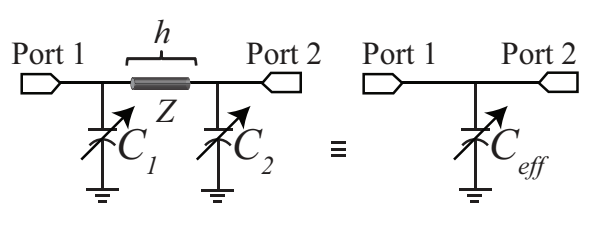

(c)

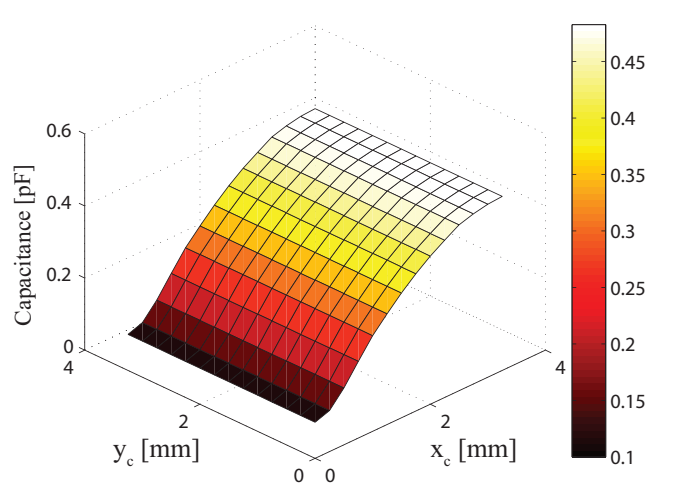

(e)

Figure 1.6 (a) The unit cells of the two capacitive layers used to synthesize a single effective capacitive layer of the MEFSS of Fig. 1(a) can overlap with each other. This overlap is modeled by the vector $\left(x_{c}, y_{c}\right)$. (b) 3D topology of the unit cell of a composite patch layer showing the offset between the capacitive patches and the dielectric slab that separates them. (c) The equivalent circuit model of the capacitive structure shown in Fig. 1.6(b). (d)-(e) The extracted effective capacitance of the capacitive structure shown in Fig. 6(b) consisting of two cascaded capacitive patches and a thin substrate separating them. The structure has the unit cell dimensions of $6.5 \mathrm{~mm} \times 6.5 \mathrm{~mm}$. The gap size for both layers is $s=0.5 \mathrm{~mm}$ and the patch size is $P=6 \mathrm{~mm}$. The thickness of the substrate is $h=0.2 \mathrm{~mm}$. The study has been done for both (d) Vertical and (e) Horizontal polarizations. 
To demonstrate the effect of offset overlapping, we have examined a periodic structure composed of two capacitive patch layers with unit cell dimensions of $D=6.5 \mathrm{~mm}$ and the gap spacing of $s=0.5 \mathrm{~mm}$. Both layers are assumed to be in free space and the spacing between them is $h=0.2 \mathrm{~mm}$. As shown in Fig. 1.6(a), the overlap between two patch layers is modeled with a vector $\left(x_{c}, y_{c}\right)$ connecting the centers of the two patches, within each unit cell of the structure, together. $x_{c}$ refers to the horizontal offset between the positions of the two patches and $y_{c}$ refers to the vertical offset between them. The structure is simulated using full-wave EM simulations in CST Studio to compute its effective capacitance as a function of the vector $\left(x_{c}, y_{c}\right)$. To do this, the unit cell of the structure shown in Fig. 1.6(b) is simulated using the periodic boundary conditions. The transmission and reflection coefficients of the structure for a normally incident wave are calculated for the two orthogonal polarizations. These include the vertical and horizontal polarizations in which the electric field is respectively oriented along the $\hat{y}$ and $\hat{x}$ directions. Subsequently, the simplified equivalent circuit model of the structure, shown in Fig. 1.6(c) (right), is simulated in a circuit simulation software (Agilent Advanced Design System) and the transmission coefficient of the equivalent circuit model is calculated. Finally, the value of $C_{e f f}$ is tuned in ADS to match the magnitudes and the phases of the transmission and reflection coefficients obtained from the full-wave simulation and the simplified circuit model together. This way, the effective capacitance is calculated.

Figs. 1.6(d)-1.6(e) show the results obtained from these case studies for the aforementioned structure for both the vertical and horizontal polarizations. For both polarizations, the capacitance value is calculated as a function of $x_{c}$ and $y_{c}$. For the vertical polarization, $y_{c}$, which represents the vertical offset between two patches, has the most significant effect on the capacitance value while $x_{c}$, which represents the horizontal offset between two patches, only has a negligible effect. However, for the horizontal polarization, $x_{c}$ has the major effect and $y_{c}$ only affects the capacitance negligibly. These can be described easily by the alignment of the direction of the electric field vector of the incident wave and the gap between two adjacent patches. Combining these two figures, we can find out that the maximum capacitance happens when the offset vector is equal to $\left(x_{c}, y_{c}\right)=(D / 2, D / 2)$. For this structure, the maximum value of the capacitance is $0.48 \mathrm{pF}$. 
The minimum capacitance value of $0.09 \mathrm{pF}$ is obtained for the case when both patches are aligned together and $\left(x_{c}, y_{c}\right)=(0,0)$.

\subsubsection{Approximate Formula for Calculating the Capacitance of Cascade Over- lapping Patches}

To better understand the impact of the offset overlap on the capacitance of the cascaded patches and facilitate the design process of such structures, we have developed an analytical formula that can be used to approximate the effective capacitance of a two layer stack of cascaded capacitive patches with the offset vector of $\left(x_{c}, y_{c}\right)=(D / 2, D / 2)$. Fig. 1.7 shows a unit cell of such a structure. Observe that four parts of the upper patch layer form effective parallel plate capacitors of $C_{c}$ with the bottom patch layer. $C_{c}$ can be easily approximated by the following formula:

$$
C_{c}=\frac{\epsilon_{\circ} \epsilon_{r_{e f f}}(D-s)^{2}}{4 h}
$$

Where $D$ is the unit cell size, $s$ is the gap size between two adjacent patches, $h$ is the thickness of the dielectric between two patch layers, and $\epsilon_{\text {eff }}$ represents the effective dielectric constant between the two layers. The total capacitance is modeled as the parallel combination of the intrinsic capacitance $C_{i}$, brought by the capacitive gap, in parallel with the series-parallel network of $C_{c}$ capacitors as shown in Fig. 1.7(b). Therefore,

$$
C_{\text {total }}=C_{c}+C_{i}
$$

where

$$
C_{i}=\epsilon_{\circ} \epsilon_{r_{e f f}} \frac{2 D}{\pi} \ln \left(\frac{1}{\sin \left(\frac{\pi s}{2 D}\right)}\right)
$$

These analytical formulas are used to calculate the effective capacitance of the two layer stack of capacitive patches examined in Section 1.1.3 as a function of the unit cell size. Fig. 1.8 shows the capacitance values calculated using (3)-(5) alongside the values extracted from full-wave EM simulations. As can be observed, both results are in good agreement. Thus, the analytical formula 
can be used in a design procedure of the proposed harmonic-free FSS to predict the capacitances to a first order approximation.

\subsection{Design Procedure and a Design Example}

\subsubsection{Design Procedure}

The design procedure of the proposed structure is based on synthesizing the desired filter response of the equivalent circuit model of Fig. 1.5(b) and mapping the equivalent circuit parameters to the physical parameters of the proposed MEFSS. All the steps are similar to the procedure described in Section 1.1.1 and also that reported in [21] except the mapping of the effective parameters of the equivalent circuit model to the geometrical parameters of the capacitive layers constituting the FSS. In the mapping step, the desired values of the capacitors and inductors obtained from the equivalent circuit model are mapped to the geometrical parameters of the proposed structure. We assume that the dielectric constant of all the substrates used in the FSS are known. For simplicity, we also assume that all substrates used in the FSS have the same dielectric constants and that the thicknesses of the substrates used in between the capacitive multilayers are also equal and known. The gap size between the sub-wavelength capacitive patches in each capacitive layer is primarily determined by the minimum feature size that can be reliably fabricated using the fabrication technology of choice. In general, the smallest gap size is chosen to ensure that the unit cell dimensions of the FSS can be reduced as much as possible. This will increase the bandwidth over which no spurious transmission window occurs. Assuming that the gap size, $s$, is known and fixed, the unit cell size, $D$, can be obtained using (3)-(5). Then, the width of the wire grids is determined from:

$$
L=\mu_{\circ} \mu_{r_{e f f}} \frac{D}{2 \pi} \ln \left(\frac{1}{\sin \left(\frac{\pi w}{2 D}\right)}\right)
$$

Due to the close proximity of the metal layers, the presence of each layer will affect the capacitance or inductance of the other layers. Therefore, physical parameters obtained using this design process 


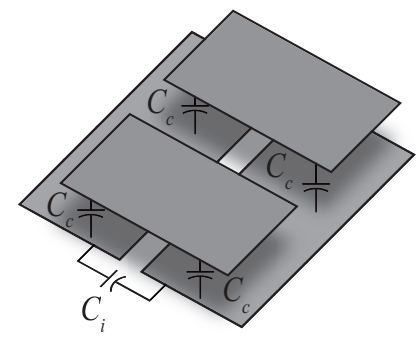

(a)

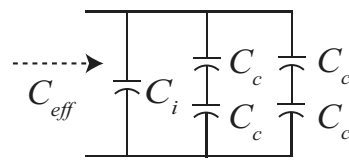

(b)

Figure 1.7 (a) The capacitive structure composed of two cascaded capacitive layers can be modeled as four capacitors with capacitance values of $C_{c}$ and one capacitor with a capacitance value of $C_{i}$ as depicted. (b) The equivalent circuit model of the capacitive structure showing the relative arrangement of $C_{c}$ and $C_{i}$ capacitors.

may need to be tuned slightly to achieve the desired response. This can be done by following an iterative procedure similar to the one described in [16] and [21] and will not be repeated here.

\subsubsection{Design Example and Simulation Results}

The procedure presented in Section 1.2.1 was followed to design an MEFSS prototype with a second-order bandpass response having a center frequency of $f_{0}=3.0 \mathrm{GHz}$, a fractional bandwidth of $\delta=20 \%$, and no spurious transmission bands up to aproximately $27 \mathrm{GHz}$. The equivalent circuit parameters of the structure (shown in Fig. 1.5(b)) are first determined following the design procedure described in [21]. In doing so, we assumed that dielectric substrates are non magnetic and have a dielectric constant of $\epsilon_{r}=2.2$ (Rogers RT/duroid 5880). A conventional second-order MEFSS (of the type shown in Fig. 1(a)) has two capacitive layers and one inductive layer as shown in Fig. 1.1. In this harmonic-suppressed MEFSS, each of the capacitive layers are implemented by using two closely-spaced, overlapping capacitive layers as described in Section 1.1.3. The physical parameters of the MEFSS are obtained using the design procedure discussed in Section 1.2.1. The structure has unit cell dimensions of $D=5.2 \mathrm{~mm}$ which is equivalent to approximately $\lambda_{0} / 20$, where $\lambda_{0}$ is the free space wavelength at $f_{0}=3.0 \mathrm{GHz}$. Since this MEFSS is composed of multiple substrates that need to be bonded together, the effect of the bonding material on the response of the MEFSS must also be taken into account. The bonding material used here is Rogers 4450F prepreg with the dielectric constant of $\epsilon_{r}=3.58$ and a thickness of $0.1 \mathrm{~mm}$. Introduction of the bonding 


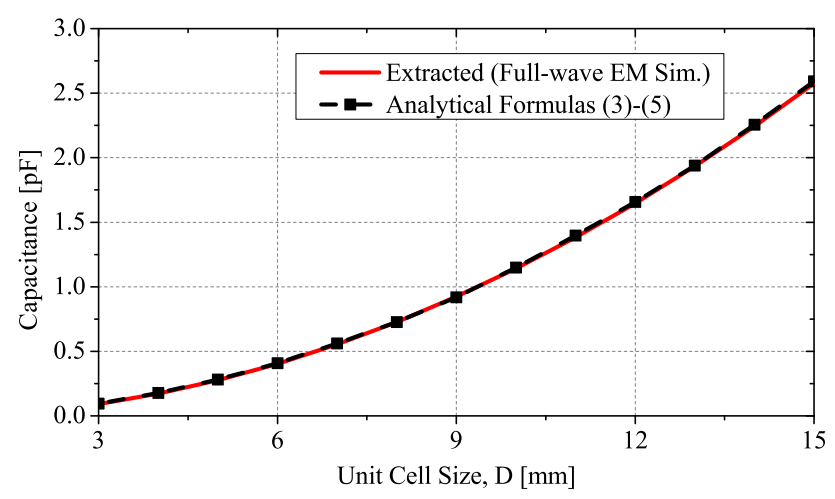

Figure 1.8 Comparison between the capacitance values predicted using the analytical method and those extracted from full-wave EM simulations. The results are shown for the structure examined in section 1.1.3.

layers creates an asymmetry in the topology of the proposed MEFSS, which slightly changes its response. This asymmetry is eliminated by using two closely-spaced wire grids on the two sides of the middle bonding layer instead of using just one wire grid on one side as shown in Fig. 1.9. Using this strategy, the two inductive layers in the middle, which are separated by a thin prepreg layer, act as a single composite inductive layer. This way, the symmetry of the structure is maintained and impact of the prepreg layers on the response of the MEFSS can be minimized. The final physical parameters of the structure are listed in Table 1.2. This structure is simulated in CST Microwave Studio and its frequency response is calculated. Fig. 1.10 shows the transmission coefficient of this FSS in the 0-27 GHz range. As can be observed, a transmission window centered at $3.0 \mathrm{GHz}$ is achieved and the FSS does not have any spurious transmission bands up to $27 \mathrm{GHz}$ as expected.

\subsection{Experimental Verification and Measurement Results}

A prototype of the aforementioned harmonic-suppressed MEFSS (discussed in Section 1.2.2) is fabricated using standard PCB lithography and substrate bonding techniques. The fabricated prototype has six metal layers, four dielectric substrates, and panel dimensions of $50 \mathrm{~cm} \times 37 \mathrm{~cm}$. Rogers RT/duroid 5880 substrates $\left(\epsilon_{r}=2.2\right)$, with the thickness of $1.575 \mathrm{~mm}$ are used between inductive layers and capacitive multilayers. The same substrate material with the thickness of $0.127 \mathrm{~mm}$ is used between two cascaded captive patches. All the dielectric substrates are bonded 


\begin{tabular}{|c||c|c|c|c|}
\hline Parameter & $D$ & $w_{1}$ & $w_{2}$ & $h_{b}$ \\
\hline Value & $5.2 \mathrm{~mm}$ & $2 \mathrm{~mm}$ & $2 \mathrm{~mm}$ & $0.101 \mathrm{~mm}$ \\
\hline Parameter & $P_{1}$ & $s_{1}$ & $P_{2}$ & $s_{2}$ \\
\hline Value & $4.85 \mathrm{~mm}$ & $0.35 \mathrm{~mm}$ & $4.85 \mathrm{~mm}$ & $0.35 \mathrm{~mm}$ \\
\hline Parameter & $h_{1}$ & $h_{2}$ & $h_{3}$ & $h_{4}$ \\
\hline Value & $1.575 \mathrm{~mm}$ & $1.575 \mathrm{~mm}$ & $0.127 \mathrm{~mm}$ & $0.127 \mathrm{~mm}$ \\
\hline
\end{tabular}

Table 1.2 Physical parameters of the $2^{\text {nd }}$-order FSS with a center frequency of $3 \mathrm{GHz}$, the fractional bandwidth of $\delta=20 \%$, and no harmonics up to $27 \mathrm{GHz}$ discussed in Section 1.2.2.

together using a $0.1 \mathrm{~mm}$ thick Rogers $4450 \mathrm{~F}$ binding film with $\epsilon_{r}=3.58$. The total thickness of the structure, including the bonding layers, is $3.7 \mathrm{~mm}$ which is less than $\lambda_{0} / 25$ at $3.0 \mathrm{GHz}$. Fig. 1.11 shows a photograph of the fabricated harmonic-suppressed MEFSS.

The measurement setup consists of a large metallic screen with the dimensions of $1.8 \mathrm{~m} \times$ $1.2 \mathrm{~m}$ with a rectangularly shaped opening having the same dimensions as those of the FSS at its center. This screen is placed between the transmitting and receiving antennas both connected to the two ports of a vector network analyzer (VNA). The large metallic fixture ensures that a transmitted EM wave must pass through the FSS to arrive at the receiver. Absorbers are used to cover points of specular reflection in the surrounding environment (e.g. on the ground and side walls of the room). Additionally, range gating in the VNA is used to eliminate the effects of scattering and diffraction of the EM waves from the edges of the metallic fixture that host the FSS as well as as well as the effects of the multiple reflections between two antennas as discussed in [16]. Transmission coefficient measurements are carried out in two steps. First, the transmission coefficient of the screen without the FSS is measured and used for calibration. Then, the FSS is placed in the opening and its transmission coefficient is measured once again. The transmission coefficient of the MEFSS is obtained using these two measurement results. Measurements have been performed in $2 \mathrm{GHz}-27.0 \mathrm{GHz}$ frequency range using multiple pairs of transmitting and receiving antennas that cover different parts of this frequency range. Over the $2 \mathrm{GHz}-8 \mathrm{GHz}$ frequency band, a pair of dual ridge horn antennas are used. A pair of X-band and a pair of K-band horn antennas are 


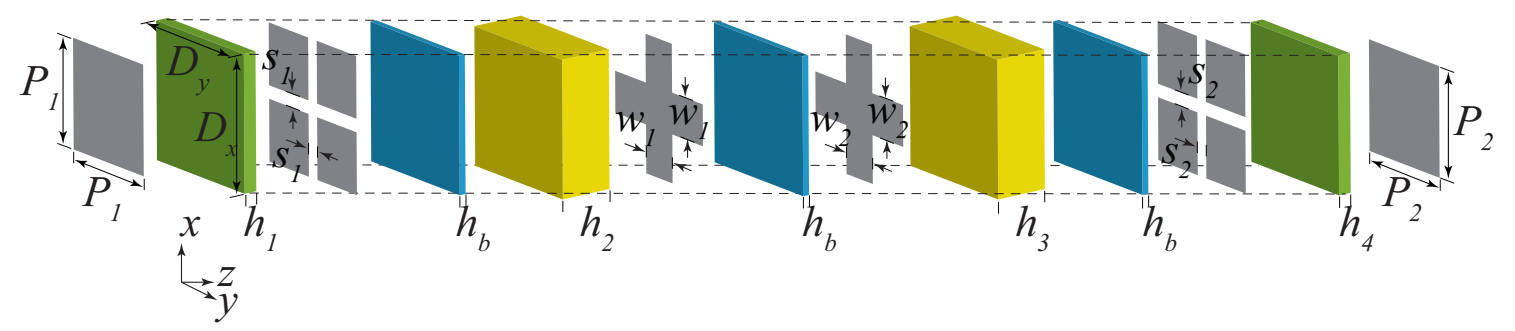

Figure 1.9 Unit cell of the proposed harmonic-suppressed $2^{\text {nd }}$-order bandpass FSS discussed in Section 1.2.2.

used to do the measurements in the $7.0 \mathrm{GHz}-15 \mathrm{GHz}$ and $15 \mathrm{GHz}-27.0 \mathrm{GHz}$ frequency bands respectively. In these measurements, the X-band and K-band horn antennas were used outside of their recommended frequency bands of operation (e.g., 7.0-8.0 GHz and 12-15 GHz for the X-band horns and $15 \mathrm{GHz}-18 \mathrm{GHz}$ for the K-band horns). This was done primarily due to the unavailability of other standard antennas that were specifically designed for operation at these bands during our measurements. Fig. 1.10 shows the measured frequency response of the MEFSS along with the full-wave simulation results. As can be observed, a relatively good agreement between the measured and simulated results is observed. The discrepancies observed between the measurement and simulation results in the vicinity of $7.0 \mathrm{GHz}$ and $15.0 \mathrm{GHz}$ are primarily attributed to using the receive and transmit antennas outside of their recommended frequency bands. Nonetheless, the measured result demonstrates clearly that the FSS does not have any spurious transmission windows up to $27.0 \mathrm{GHz}$.

The response of the fabricated prototype is also measured for oblique incidence angles for the TE and TM polarizations of incidence. Fig. 1.12(a) shows the transmission coefficients of the structure measured for the TE polarization for incidence angles in the range of $0^{\circ}-60^{\circ}$. As can be observed, the FSS response is stable for such large incidence angles. Additionally, the structure maintains its harmonic suppressed operation for the TE polarization up to $27.0 \mathrm{GHz}$ for incidence angles in the range of $\pm 60^{\circ}$. Fig. 1.12(b) shows the measured transmission coefficients of the structure for the TM polarization of incidence in the range of $0^{\circ}-60^{\circ}$. Similar to the previous case, the FSS maintains its second-order bandpass response. However, the center frequency of operation of the structure shifts higher than $3.0 \mathrm{GHz}$ for incidence angles exceeding $45^{\circ}$. This is 


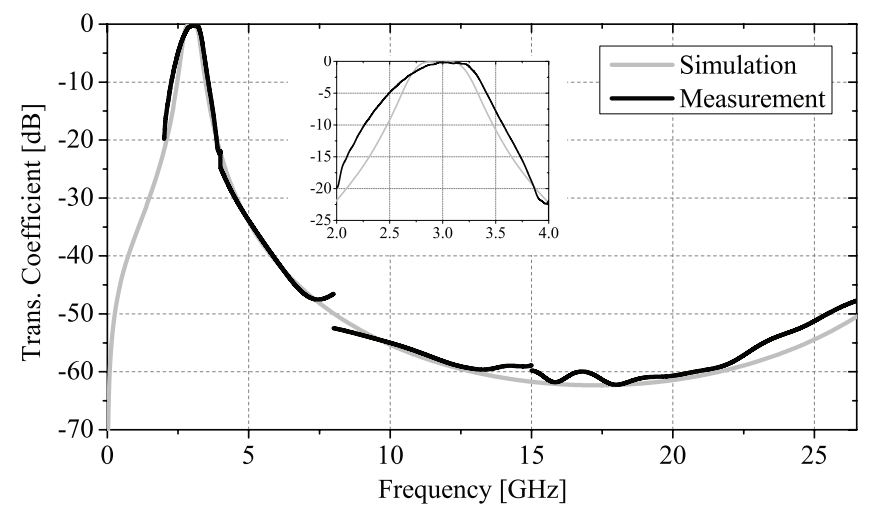

Figure 1.10 Measured and calculated transmission coefficients of the FSS prototype discussed in Section 1.2.2 and shown in Fig. 1.11. The MEFSS is composed of the unit cells shown in Fig. 1.9 with physical dimensions reported in Table 1.2.

also observed in other MEFSSs of the type shown in Fig. 1 (e.g., see [16]). This behavior can be explained by examining the variations of the parameters of the equivalent circuit model of the FSS under oblique incidence angles. Specifically, for the TE polarization of incidence, the capacitance values of the patch layers decrease as the angle of incidence increases while the inductance value of the wire grid does not change [27]. For the TE polarization, the series inductances - associated with small transmission lines representing the dielectric substrates of the MEFSS - increase as the incidence angle increases. These two effects compensate each other resulting in a stable center frequency of operation as the angle of incidence changes. For the TM polarization, however, the inductance of the wire grid in the middle layer decreases as the incidence angle increases while the capacitance values of the patch layers do not change [27]. The values of the series inductances representing the short transmission lines modeling the dielectric substrates, however, decrease as the angle of incidence increases. The reduction of these inductance values results in increasing the center frequency of operation of the MEFSS for the TM polarization as the incidence angle increases. This effect, however, become significant only when the incidence angle increases beyond $45^{\circ}$ as seen in Fig. 1.12. Additionally, for the TM polarization, as the angle of incidence is increased, one of the higher spurious transmission windows starts to shift towards lower frequencies and is observed in the results shown in Fig. 13(b) for $\theta=45^{\circ}$ and $\theta=60^{\circ}$. The peak transmission coefficient of this spurious band, however, remains below -20 dB for incidence angles up to 

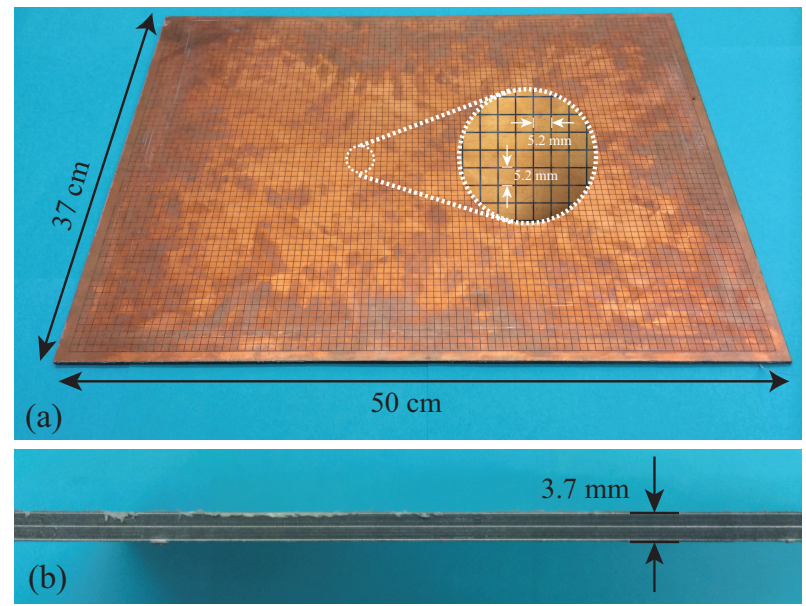

Figure 1.11 (a) Photograph of the fabricated harmonic-suppressed MEFSS prototype. (b) Side view of the fabricated prototype.

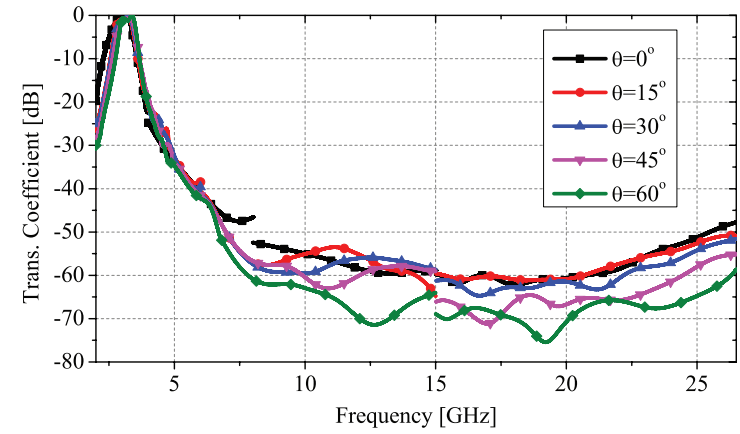

(a)

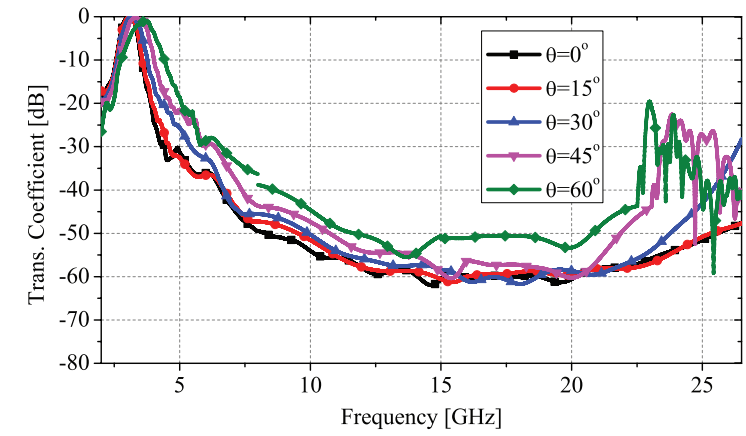

(b)

Figure 1.12 (a)-(b) Measured transmission coefficients of the fabricated harmonic-suppressed MEFSS prototype discussed in Section 1.2.2 and shown in Fig. 1.11 for oblique incidence angles for the (a) TE and (b) TM polarization of incidence. 
$60^{\circ}$. Thus, the MEFSS maintains its desired harmonically-suppressed transfer function for the TM polarization for incidence angles in the $\pm 60^{\circ}$ range as well.

\subsection{Conclusions}

We presented a new method for designing miniaturized-element frequency selective surfaces with higher-order bandpass responses, which are free of spurious transmission windows over an extremely wide frequency band. The proposed harmonic-suppressed MEFSSs take advantage of the concept of cascaded, overlapping capacitive patch layers to drastically reduce the unit cell size of the structure. Using multiple closely-spaced capacitive layers with overlapping unit cells to synthesize a single effective capacitive layer leads to a larger capacitance value for given unit cell dimensions. As a result, the natural resonant frequencies of these patches and the apertures within the wire grids shift to higher frequencies and a harmonic-free response over an extremely large bandwidth can be achieved. The proposed concept was experimentally verified by designing an MEFSS prototype having a second-order bandpass response with a fractional bandwidth of $20 \%$ at $3.0 \mathrm{GHz}$, which does not demonstrate any spurious transmission windows up to $27 \mathrm{GHz}$. This MEFSS was also fabricated and experimentally characterized using a free-space measurement setup. Measurements confirmed that the fabricated structure is indeed free of spurious transmission windows over the expected frequency band of operation. Additionally, we examined the performance of this prototype for various incidence angles and polarizations of the incident EM wave. The fabricated MEFSS prototype demonstrates a stable frequency response for both the TE and TM polarizations of incidence and maintains its harmonic free operation for incidence angles in the $\pm 60^{\circ}$ range for both the TE and TM polarizations of incidence. 


\section{LIST OF REFERENCES}

[1] B. A. Munk, Frequency Selective Surfaces: Theory and Design. New York: Wiley-Interscience, 2000.

[2] N. Behdad, "A second-order band-pass frequency selective surface using non-resonant subwavelength periodic structures," Microwave and Optical Technology Letters, vol. 50, issue 6, pp. 1639-1643, June 2008.

[3] S. Chakravarty, R. Mittra, and N. R. Williams, "On the application of the microgenetic algorithm to the design of broad-band microwave absorbers comprising frequency-selective surfaces embedded in multilayered dielectric media," IEEE Trans. Microw. Theory Tech., vol. 49, no. 6, pt. 1, pp. 1050-1059, 2001.

[4] A. Fallahi, A. Yahaghi, H. Benedickter, H. Abiri, M. Shahabadi, and C. Hafner, "Thin wideband radar absorbers," IEEE Trans. Antennas Propag., vol. 58, no. 12, pp. 4051-4058, 2010.

[5] A. Monorchio, G. Manara, and L. Lanuzza, "Synthesis of artificial magnetic conductors by using multilayered frequency selective surfaces," IEEE Antennas Wireless Propag. Lett., vol. 1, no. 1, pp. 196-199, 2002.

[6] M. A. Hiranandani, A. B. Yakovlev, and A. A. Kishk, "Artificial magnetic conductors realised by frequency-selective surfaces on a grounded dielectric slab for antenna applications," IEE Proc. Microw. Antennas Propag., vol. 153, no. 5, pp. 487-493, 2006.

[7] M. Al-Joumayly and N. Behdad, "Wideband planar microwave lenses using sub-wavelength spatial phase shifters," IEEE Trans. Antennas Propag., vol. 59, no. 12, pp. 4542-4552, Dec. 2011.

[8] M. Li, M. Al-Joumayly, and N. Behdad, "Broadband true-time-delay microwave lenses based on miniaturized element frequency selective surfaces," IEEE Trans. Antennas Propag., vol. 61, no. 3, pp. 1166-1179, March 2013.

[9] J.A. Encinar, "Design of two-layer printed reflectarrays using patches of variable size," IEEE Trans. Antennas Propag., vol. 49, no. 10, pp. 1403-1410, Oct 2001. 
[10] W. S. Arceneaux, R. D. Akins, and W. B. May, “Absorptive/Transmissive Radome," U.S. Patent 5,400,043, May 21, 1995.

[11] B. Li and Z. Shen, "Synthesis of quasi-elliptic bandpass frequency-selective surface using cascaded loop arrays," IEEE Trans. Antennas Propag., vol. 61, no. 6, pp. 3053-3059, June 2013.

[12] F. Costa and A. Monorchio, "A frequency selective radome with wideband absorbing properties,” IEEE Trans. Antennas Propag., vol. 60, no. 6, pp. 2740-2747, June 2012.

[13] K. Sarabandi and N. Behdad, "A frequency selective surface with miniaturized elements," IEEE Trans. Antennas Propag. , vol. 55, no. 5, pp. 1239-1245, May 2007.

[14] H. Liu, K. L. Ford, and R. J. Langley, "Miniaturised bandpass frequency selective surface with lumped components, Electron. Lett., vol. 44, no. 18, pp. 1054-1055, Aug. 28, 2008.

[15] F. Bayatpur and K. Sarabandi, "Multipole spatial filters using metamaterial-based miniaturized-element frequency selective surfaces," IEEE Transactions on Microwave Theory and Techniques, vol.56, no. 12, pp. 2742-2747, Dec. 2008.

[16] N. Behdad and M. Al-Joumayly, "A new technique for design of lowprofile, second-order, band-pass frequency selective surfaces," IEEE Trans. Antennas Propag., vol. 57, no. 2, pp. 452-459, Feb. 2009.

[17] F. Bayatpur and K. Sarabandi, "Tuning performance of metamaterial-based frequency selective surfaces," IEEE Transactions on Antennas and Propagation, vol. 57, no. 2, pp. 590-592, Feb. 2009.

[18] R. J. Langley, L. L. Hui, and K. L. Ford, "Design methodology for a miniaturized frequency selective surface using lumped reactive components, IEEE Trans. Antennas Propag., vol. 57, no. 9, pp. 2732-2738, Sep. 2009.

[19] C.-N. Chiu and K.-P. Chang, "A novel miniaturized-element frequency selective surface having a stable resonance, IEEE Antennas Wireless Propag. Lett., vol. 8, pp. 1175-1177, 2009.

[20] N. Behdad and M. Al-Joumayly, "A generalized synthesis procedure for low-profile frequency selective surfaces with odd-order band-pass responses," IEEE Trans. Antennas Propag., vol. 58, No. 7, pp. 2460-2464, July 2010.

[21] M. Al-Joumayly and N. Behdad, "A generalized method for synthesizing low-profile, bandpass frequency selective surfaces with nonresonant constituting elements," IEEE Trans. Antennas Propag., vol. 58, no. 12, pp. 4033-4041, Dec. 2010.

[22] Guohui Yang, Tong Zhang, Wanlu Li, and Qun Wu, "A novel stable miniaturized frequency selective surface," IEEE Antennas and Wireless Propagation Letters, vol.9, pp.1018-1021, 2010. 
[23] Cheng-Nan Chiu and Wen-Yi Wang, "A dual-frequency miniaturized-element FSS with closely located resonances," IEEE Antennas and Wireless Propagation Letters, vol. 12, pp.163$165,2013$.

[24] Feng Deng, XueQin Yi, and WeiJun Wu, "Design and performance of a double-layer miniaturized-element frequency selective surface," IEEE Antennas and Wireless Propagation Letters, vol. 12, pp.721-724, 2013.

[25] M. Li and N. Behdad, "Fluidically tunable frequencyselective/phase shifting surfaces for high-power microwave applications," IEEE Trans. Antennas Propag., Vol. 60, no. 6, pp. 27482759, June 2012.

[26] M. Li and N. Behdad, "Frequency selective surfaces for pulsed high-power microwave applications," IEEE Trans. Antennas Propag., vol. 61, no. 2, pp. 677-687, Feb. 2013.

[27] O. Luukkonen, C. Simovski, G. Granet, G. Goussetis, D. Lioubtchenko, A. V. Raisanen, and S. A. Tretyakov, "Simple and accurate analytical model of planar grids and high-impedance surfaces comprising metal strips or patches," IEEE Transactions on Antennas and Propagation, vol. 56, no.6, pp. 1624-1632, June 2008. 